\title{
Building a Collaborative Network for Education and Training in International Trade Facilitation Clusters
}

\author{
John A. Clendenin ${ }^{1}$, Nadya N. Petrova ${ }^{2}$, and Joshua K. Gill ${ }^{3}$ \\ ${ }^{1}$ Instituto de Empressa \\ Sportaprofesor.ie.edu \\ ${ }^{2}$ Supply Chain Center of Regional Excellence \\ Nadya@ICLogistics.com \\ ${ }^{3}$ Inner Circle Logistics Inc. \\ jgill@ICLogistics.com
}

\begin{abstract}
The authors present the benefits of collaboration rather than competition in developing educational and training resources for international trade within a geographic region and explore the challenges for business partners, governments and educational institutions. The paper indicates that flexibility in the 21 st Century is critical, particularly when striving for virtual implementations of the solution services. It is essential, say the authors, for educators, governments and business executives to focus on performance and the careful orchestration and integration of business, policy and information technology for "Networking" that successfully stimulates inter-governmental cooperation and innovative policies that foster Regional trade facilitation. An innovative way to enhance 21st Century Trade Facilitation is offered with Supply Chain Centers of Regional Excellence (SCcORE).
\end{abstract}

Keywords: Collaborative Networks for Education, Trade Facilitation, clustering, B2G, B2B, e-infrastructure.

\section{Introduction}

The benefits of collaboration rather than competition in developing international trade within a geographic region are clear. These benefits include; improved performance among supply chain partners, lower costs, energy conservation and reduced congestion along transportation corridors [1]. However, challenges exist such as the need for training, formal education, and outside consulting services that require careful planning business partners, governments and educational institutions. When developing lifelong learning methodologies in the $21^{\text {st }}$ Century flexibility is crucial, particularly when striving for virtual implementations of the solution services. Both trainees and educational organizations face the challenges of combining time, needs, and resources in a complex system of systems that often have divergent priorities. This complex, interrelated process involving multiple actors was described by Porter as the evolution of economic clusters linked by common technology and skills that are an indelible part of today's knowledge based economy [2]. 


\section{Collaborative Networks}

\subsection{General}

Collaborative networks in education can lead the way to professional training programs adapted to the needs of the local business community through a virtual exchange of academic competencies. It is essential for educators, governments and business executives to focus on performance and the careful orchestration and integration of business, policy and information technology. The authors address these three pillars: academia, government, business community (chambers of commerce) and the collaboration required for successful "Networking" for education and training which stimulates inter-governmental cooperation and innovative policies that foster Regional trade facilitation.

\subsection{Innovation}

The evolution of innovation and impact of net-centric tools has been a substantive force in the collaborative capabilities of networks that create economic possibilities that defy traditional molds and allow for the formation of collaborative networks over increasing geographic distance [3], [4]. Successful collaboration, rather than competition, to enhance 21st Century Trade Facilitation is made possible by Supply Chain Centers of Regional Excellence (SCcORE). These "Centers of Excellence" represent an innovative method to stimulate the free movement of knowledge in an effective Regional "Cluster" for increased competence and competitive advantage. SCcORE enables a geographic Region such as Southeastern Europe (SEE) (is SEE not more "accepted" these days?) to compete as a "Trade Cluster" sharing the resources and competitive advantages of collaboration. Today several examples exist of efforts to foster the development of trans-boundary clusters utilizing the connective effects of ICT including Western Europe, the Baltic States, and Central Europe [5], [6], [7].

\section{Networks for Trade Facilitation Clusters}

\subsection{Rapid Change and ICT Infrastructure}

Information technology (IT) has become an integral part of our life with new developments occurring at pace never before seen, bringing with it rapid changes in our personal and professional worlds. These changes range from daily interpersonal communication through bio-modified interference to innovative business models that quickly become an indelible part of today's economics and politics. Trade facilitation is a powerful tool for boosting economic growth especially in countries with strategic geographic location. In the past, trade flourished in regions found at geographic "choke points" along trade corridors. An example of this can be found on the Balkan Peninsula, located on trade routes of the Silk Road and an important staging point for trade between Europe and Asia. However, today's prerequisites for the development of global trade are not only geographical resources and geopolitical stability but also efficiency and capabilities in facilitating trade. For example, the importance of utilizing innovative technologies within knowledge networks for trade cluster development 
has been demonstrated in the Strait of Mallacca. Knowledge transfer was a crucial factor in the emergence of knowledge clusters across four distinct trade clusters: Northwest Malaysian, West Malaysian, North Sumatra, and Singapore-Johore. The use of technology was fundamental in producing innovation, promoting knowledge sharing, and engaging in knowledge transfer to stakeholders through education and training [8]. For example, modern trade facilitation is predicated on building physical infrastructure to facilitate the movement of goods while simultaneously developing an interconnected banking system to facilitate the movement of money. This "developmental mix" to move goods more efficiently along trade corridors is predicated on ICT infrastructure and innovations in net-centric connectivity.

\subsection{Facilitating Trade}

The efficient and speedy movement of information requires e-infrastructures to facilitate the timely exchange of trade documents among border agencies and trading partners. In an economic environment defined by global trade, the region that is capable of building an e-infrastructure and integrating its transportation and cross-border services into the global supply chain has a distinct competitive advantage. As integrated ICT based trade facilitation continues to be adopted globally, the flow of information and goods will follow the path of least resistance and migrate to the trade corridors that are ICT enabled and offer lower costs, improved shipment visibility, and lower risk [9]. For example, the World Bank consistently ranks Singapore ahead of the Netherlands, Germany, Japan, Hong Kong, and China as a hub for global trade due to its world-class infrastructure and excellent connectivity.

Pervasive in the private sector, ICT is a prerequisite to survive that must be embraced, not only in policy making but also, in educational systems. The benefits of integrating services along an e-enabled trade corridor accrue to participating countries, the business community, and private citizens. The challenge is acquiring the valuable knowledge and skills to become a productive actor in an integrated network of education and life-long learning made up of business, policy and technology with the core philosophy of innovation through collaboration. Core challenges to the creation of such a network is incorporation of the collective learning of multi-disciplinary knowledge for improved business processes, clear understanding of the global marketplace, technical understanding required for continued innovative capacity, adherence to norms valued in a Network Economy and the importance of interaction with and trust of the local community for continued support [10].

A single educational organization is limited in providing knowledge to its individual network but, a system of interconnected universities and educational centers within a region shares an increased capacity as the power of individual networks interact. This System-of-Systems (SoS) focuses on core, common, and unique attributes within the system and its members to ensure mutual exclusivity while being collectively exhaustive [13].

As Kevin Kelly cites Larry Keely in the book New Rules for the New Economies, "No one is as smart as everyone" [11]. Collaborating in a network, they are able to share competencies in a field by sharing best practices, exchanging lectors, bringing academicians together to optimize academic research, exchanging students, and providing academic internships. The collaborative opportunities among educational organizations are abundant as long as the combined competences compliment each other 
in a field and their sharing increases the value to all. The basic principle of network economic theories includes the concept of an installed base of nodes where "as the number of nodes in a network increases arithmetically, the value of the network increases exponentially" [11]. In the educational concept, collaborating academic institutes and educational centers enjoy exponential growth of opportunities with every single addition of a new collaborating partner as more diverse and customized programs are offered to the pool of current and perspective trainees by an organization within the network. Furthermore, collaborating with partners with complementary competences or specializing in a subject strengthens members within a network as the value of the network increases with network expansion. These results have been shown to hold with continued decreases in the costs of technology that result from the innovative capacity of networks and their ability to disseminate information [13]. The synergistic impact of this approach is further explained in Figure 1.

The Internet provides the opportunity to create a semantic community using netcentric tools to foster collaboration and partnerships where information access is achieved in an integrated environment for informed decision-making based on a multidisciplinary approach [12]. Robust statistical tools and performance metrics are optimized to ensure maximum quality control and service provision for a system of regional training units that allow for the analysis of multiple networks simultaneously in which, there are multiple types of nodes (multi-node) and multiple types of links (multi-plex).

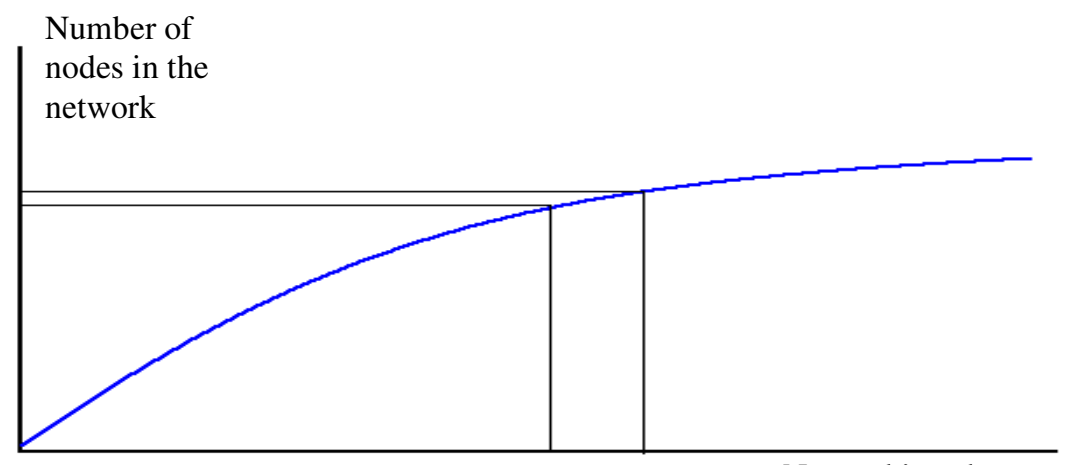

Network's value to trainees

Fig. 1. The Network Effect within a collaborative network

\subsection{Collaborative Networks}

In collaborative networks for education and lifelong learning, bringing together multiple tiers from institutes of higher education, training centers, and the public and private sector further maximizes network output by providing unprecedented reach and impact to diverse audiences.

\subsection{Life-Long Learning}

One of the growing components of academic services is the notion of life-long learning and the provision of continuing education to the community. A university can 
offer a seminar in collaboration with a network participant such as a training center that is practice-oriented rather than theory-oriented and already specializes in the needed field or profession. Collaborating in a network for education in an international environment or a large region, the training center has the capabilities to offer its products and services beyond its existing market. A local partner, as a university located in a new market, is a good channel for information distribution, training promotion, and recruitment as the university gains the capability to offer educational opportunities to an enlarged target group. Combining complimentary competences, the collaborating partners do not deprive each other of applicants for targeting different audiences. The greatest attribute of a collaborative network for education and training is the social organization of its members, integrated and supported by the local community, where the flow of information across a virtually limitless number of areas and interests is encouraged.

\subsection{Flexibility}

The importance of a collaborative network for education and lifelong learning is not only the co-existence of its constituent parts but also its flexibility in attracting external partners in maximizing the value of networking to all participants. According to Kevin Kelly, "More complex objects and services are capable of permeating far more systems and networks, thus greatly boosting their own value and the plentiful value of all the systems they touch." [11] Supporting economic growth along a trade corridor by transferring competences, a collaborative network for education thrives as the link between policy requirements and business adaptability. In order to contribute to trade facilitation effectively, it must be able to provide up-to-date programs and trainings on at various levels of professional development, as well as to consult government agencies on innovative practices and solutions.

\section{Developing and Building a Center of Excellence}

\subsection{Objectives of a Collaborative Network Program}

One of the primary objectives of building a collaborative network for education and training within a cluster is the enhanced understanding of the relationship-building process. Obtaining the ability to identify and analyze a supply chain's functions, processes and activities, both intra-firm and across supply chain partners, that add cost and cost to service trade-offs can be more effectively placed in context with the policy and theory of government and academia. This is an oft missing piece of traditional business training programs. Collaboration helps in developing an understanding of the role of managing supply chain costs and achieving the ability to identify specific customer service needs that will provide a differential advantage in the marketplace. The partnership approach allows for a more thorough understanding of supply chain system components and their complex relationships within individual companies, including cost to service trade-offs, so that a variety of analytical tools, skills and techniques can be used to solve supply chain problems and enhance the profitability of cross border operations. The understanding of the role of supply chain management in both business and government environments also helps the academic community use analytical tools, 
skills and techniques to solve logistical problems and identify research opportunities. The combination of practical and theoretical research developed in conjunction with government policy makers and business partners enables faculty members to gain the insight to apply innovative technologies and practices to further the research of supply chain optimization. Overall the objective is to position the Center of Excellence with the comprehensive ability to identify and disseminate core knowledge for successful management of global supply chains with the understanding of emerging and world class innovative technologies as they relate to various supply chain components.

\subsection{Solution Supply Chain Center of Regional Excellence (SCcORE)}

The Supply Chain Center of Regional Excellence (SCcORE) is a non-profit association developed on the "Center of Excellence" model described earlier. With a strong advisory board of business executives, professors and government leaders the SCcORE Centers are partnering with educational institutions, Chambers of Commerce and Trade Associations in the Balkans to optimize the learning and best practices involved in effective cross border trade. Currently, the Center is striving to support the development of Pan-European Trade Corridor X beginning at the Port of Thessaloniki crossing Northern Greece, the Former Yugoslav Republic of Macedonia, and Serbia through Central and Western Europe.

Combining competitive benchmarking through in-depth research in Supply Chain Management, SCcORE provides innovative ideas in Net-Centric trade facilitation. Bringing innovative ideas to policy makers and business associations, its collaborative network enriches with opportunities to provide further trainings to state agencies and businesses for competitiveness and efficiency on the global market [14]. Aiming at regional development through innovation, SCcORE brings together educational organizations (universities, educational centers, etc.) to collaborate with government agencies (ICT agency, Ministry of Transport, Ministry of State Administration, Customs, Phyto-sanitary Agency, Border Police, etc.), and business community (trade associations, chambers of commerce, etc.). Thus, the extended network is able to observe, analyze, and react multi-dimensionally to on-going trade concerns. Figure 2 describes the complex linkages within a SoS that defines the SCcORE approach.

The connection between education and policy making is vital but business communities also need to be exposed to the benefits of the developing technology to stimulate economic growth. Collaborating with business associations, a collaborative network for education and lifelong learning has a better understanding of the business needs. More important than not, it is more flexible in offering trainings and seminars to provide specific professional skills and knowledge than mainstream education. A collaborative network evolves to meet the requirements of the global environment by promoting its most suitable members and bringing value to the system through collective dissemination of competences. In the case of a trans-boundary network, SCcORE becomes vital to the "constructed advantage" through the ability to receive, absorb, and transmit innovative strategies, technological advancement and knowledge over long distances while acting as a repository of this shared knowledge that serves to enhance network value for new entrants [15]. SCcORE's capability to provide linkages addresses several development factors identified in the success of Western European high-tech clusters. Primary among these are the collective learning of 
multi-disciplinary knowledge for improved business processes, clear understanding of the global marketplace, technical understanding required for continued innovative capacity, adherence to norms valued in a Network Economy and the importance of interaction with and trust of the local community for continued support [4].

This is accomplished by linking the three pillars vital for the sustainability of economic growth: academia, government, and business. Their capability in reacting cohesively to business change and global competition shapes regional competiveness. A big obstacle both to employers and recent graduates in SEE is the incongruence between the demand and supply of labor competences. Bringing equilibrium to the job market is not a two-dimensional equation, however. The third dimension - policy - is as integral a part of the solution matrix. Policy, shaping the educational system and modeling the trade requirements, needs more transparency and ensure access to current and relevant trade statistics in order to evaluate and facilitate the overlapping of academia and business. For example, developing a trade corridor through integration of information systems requires new rules and regulations for cross-border trade but, their effectiveness depends on the way policy makers, business associations, and educational organizations communicate each others' needs in reaching the common goal.

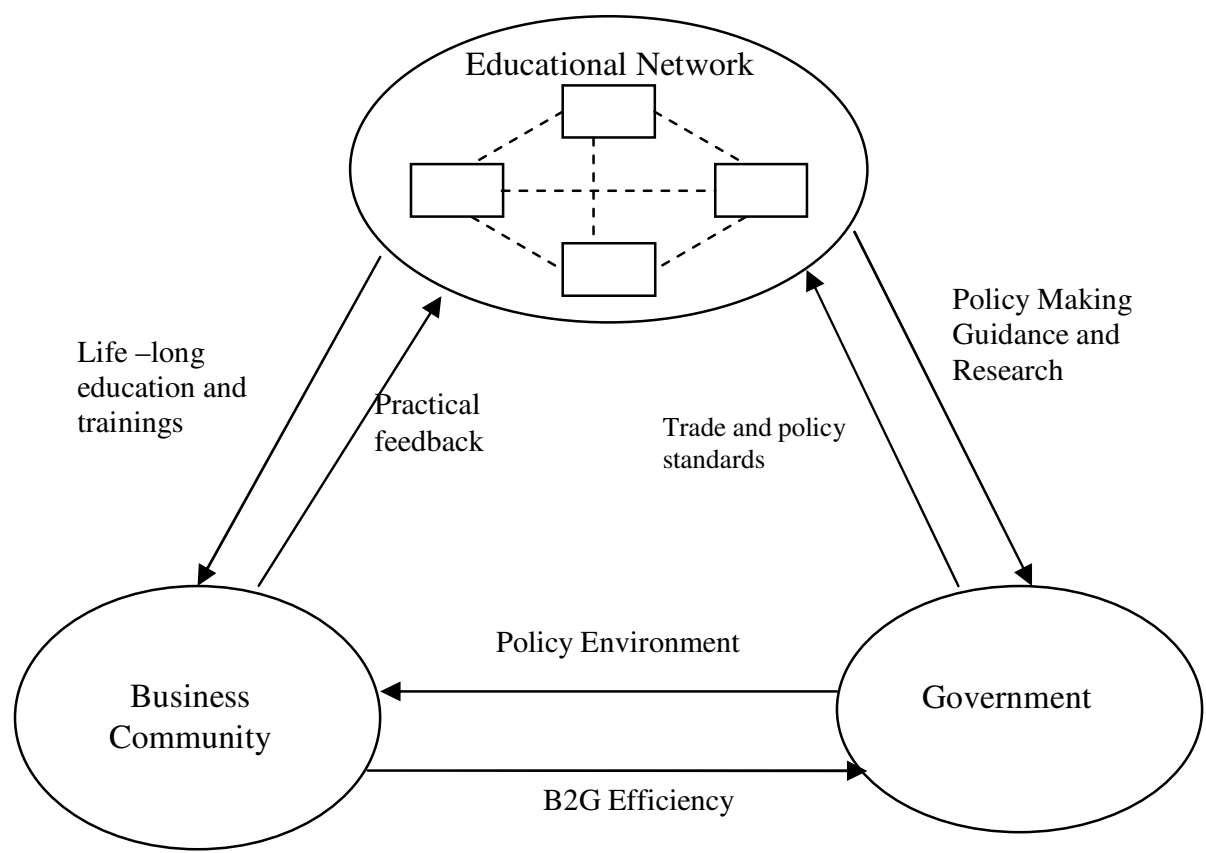

Fig. 2. Collaborative network for education in international trade facilitation: The SCcORE Model

\subsection{Knowledge Adaptation Principles}

The knowledge flow among the three pillars - government, academia, and business community serves as a generator of ideas where new products, business models, and 
opportunities proliferate. Differing from an incubator, a collaborative network for education engages a broad spectrum of organizations in achieving an adaptive and agile management and organizational culture for business and governments. The basic framework of a virtual education and training network with the vision of supporting regional competitiveness through trade facilitation provides four main principles for success [16]. The first one is Be ready for business change and global competition in a collaborative environment where optimization of processes and high connectivity among players increases their productivity. The second principle is Become technically and tactically proficient - in utilizing Internet and information systems integration and deployment among trading partners and across multiple government agencies to support the sustainability and development of the collaborative network. Real-time exchange of trade documents and data among government agencies and trading partners, timely communication among educational organizations in adapting to the business needs, and a synchronized coordination of activities among the three pillars supported by ICT integration provides a competitive edge in regional trade facilitation. The third principle for success in regional competitiveness is Benchmark for quality effectiveness - by adopting recognized benchmarking techniques as a competitive strategy for the development of pan-European trade corridors. Finally, Balance costs, inventory, and customer service - to create value by striking the right balance between business productivity and international trading procedures.

\subsection{Conclusion}

The future holds exciting prospects for partners in collaborative networks for education. A key component for success is the unlimited flow of communication and profound understanding of your partner's capabilities. With clear objectives and the connectivity of the Internet, peer-based networks will be able to do far more than expected with only minimal oversight. "Silicon Valley's greatest 'product' is the social organization of its companies and, most important, the networked architecture of the region itself - the tangled web of former jobs, intimate colleagues, information leakage from one firm to the next, rapid company life cycles, and agile email culture" [11]. This opportunity is especially appropriate for the development of e-enabled trade "clusters." The " $21^{\text {st }}$ Century Silk Road" being developed for connectivity between Pan-European Corridors and the global supply chain will bring extraordinary possibilities for the region. One example of innovation that has been demonstrated is a web-based "single-window" that allows for business-to-business (B2B), business-togovernment (B2G), and government-to-government (G2G) connectivity through seamless integration of multiple languages, technical formats, and documentation [17].

The collaborative network of education and training that strives to improve abilities, skills, and knowledge along a trade corridor holds an important role for successful development of these "Clusters." A collaborative network for education and lifelong learning cannot be restrained to educational organizations only. The flow of knowledge and proper communication among governmental decision-makers, educators, and traders is essential. The flow of knowledge is part of the movement of information, goods and payments in a global supply chain that facilitates trade. This "Cluster" is dependent on the same e-infrastructure as the three pillars of academia, government, and the business community that can provide the essential knowledge and technology transfer to facilitate competitiveness. 


\section{References}

1. Bates, W., van Opstal, D.: Five for the Future. Council of Competitiveness (2007)

2. Porter, M.E.: The Competitive Advantage of Nations. Simon and Schuster, New York (1990)

3. Porter, M.E.: Clusters of Innovation: Regional Foundations of U.S. Competitiveness. Council on Competitiveness, Washington (2001)

4. Ketels, C.: The Development of the Cluster Concept - Present Experiences and Further Developments. In: NRW Conference on Clusters, Duisburg, Germany, December 5 (2003)

5. European Biotechnology Science and Industry News. Lyonbiopole and Biowin plan to form a transborder cluster,

http: / / www . eurobiotechnews.eu/western-europe

6. The State Agency of Ukraine for Investments and Innovations: First Transborder Innovation Cluster Founded in Volyn and Rivne Regions, http: / / www. in.gov . ua

7. European Union Neighbourhood Programme. Riga, Latvia: INTERREG IIIB Baltic Sea Region, http: / / www.bsrinterreg3a.net

8. Evers, H.-D.: Knowledge hubs and knowledge clusters: Designing a knowledge architecture for development. Center for Development Research (2008)

9. Clendenin, J.A., Gill, J.K.: Increasing SME Competitiveness Using Web-based Technologies for Trade Facilitation. In: COSMO Small Business Conference (2008)

10. Keeble, D., Wilkinson, F.: Collective Learning and Knowledge Development in the Evolution of Regional Clusters of High Technology SMEs in Europe. Reg. Studies 1998 33(4), 295-303 (1998)

11. Kelly, K.: New Rules for the Network Economy, http: / / www . kk . org

12. Clendenin, J.: Complex Systems and the Science of Net-Centric Supply Chains. Invited Paper, 4th Balkan Conference in Informatics (2009)

13. Clendenin, J.A., Gill, J.K.: Towards an Integrated Southeastern European Trade Facilitation Cluster. Invited Paper, ICEIRD Conference (2009)

14. Clendenin, J.A., Petrova, N.N., Gill, J.K.: Supply Chains Centers for Regional Excellence: Repositories of Knowledge, Tools, and Training. Cosmo Small Business Conference (2008)

15. Cooke, P., Leydesdorff, L.: Regional Development in the Knowledge-Based Economy: The Construction of Advantage. J. of Tech. Transfer 31(1), 5-15 (2006)

16. Clendenin, J.: Supply Chain 101. Harvard Business School (1999)

17. Inner Circle Logistics: Facilitating Regional Competitiveness: The Southeastern Europe Project. USTDA Final Report (2008) 